\title{
Design and numerical analysis of a compound hydrocyclone
}

\author{
Qi-Dong CHEN ${ }^{1}$, Yue-Gao SUN² \\ ${ }^{1}$ Changshu institute of Technology, Changshu, 215500 \\ ${ }^{2}$ Soochow University, Suzhou, 215000
}

\begin{abstract}
The paper determines the structure of the compound hydrocyclone, by using RNG $k-\varepsilon$ model for calculation of flow distributor. By utilizing Reynolds Stress Model (RSM) and Discrete Phase Model (DPM), the internal flow fields of the hydrocyclone with different tangents of inlet channels are analyzed and the separation efficiency of the hydrocyclone is calculated. Determining the structure of flow distributor; getting the optimal angle between beveling hydrocyclone with single inlet channel and external surface of ring pipes of distributor; the best beveling angle of inlet channel of beveling hydrocyclone with single inlet channel is $5^{\circ}$.

KEYWORD: flow distributor; Reynolds Stress Model; compound hydrocyclone; Reynolds Stress Model; separation efficiency

\section{INTRODUCTION}

At present, the hydrocyclone has been widely used in many fields, such as paper, coal, mineral processing, food, petroleum, chemical industry and so on. In the basic flow of urban sewage processing, sewage contains $1.2 \%$ to $2 \%$ of solid sludge before sludge dewatering. If the sewage is further concentrated before dewatering, working efficiency of filter press or centrifuge in sludge dewatering is obviously improved and the cost of sewage processing is greatly reduced.

Experts and scholars have done lots of research on the effects of different geometric parameters and operating parameters to the performance of hydrocyclone .The influence of the pressure field and velocity field of hydrocyclone was studied by Jiang Minghu et $\mathrm{al}^{[1]}$. Wang Zunce et $\mathrm{al}^{[2]}$ studied the influence of different inlet structure parameters of the hydrocyclone on the pressure loss. The flow field of the hydrocyclone was studied by Chen Qidong et $\mathrm{al}^{[3]}$. The influence of operation parameters on the separation performance of hydrocyclone has also been studied and tested by many experts and scholars ${ }^{[4]}$. However, researches on compound hydrocyclone are relatively less. In sludge processing, since little difference lies between sludge and water, few choose to use hydrocyclone and their processing volumes also fail to meet industrial requirements. Therefore, it is necessary to design a compound hydrocyclone to improve capacity of hydrocyclone, reduce pressure loss and enhance separation efficiency.
\end{abstract}

\section{GOVERNING EQUATION}

2.1 Continuity equation and momentum equation

$$
\begin{gathered}
\frac{\partial u_{i}}{\partial x_{i}}=0 \\
\rho\left(\frac{\partial u_{i}}{\partial t}+u_{j} \frac{\partial u_{i}}{\partial x_{j}}\right)=\frac{\partial p}{\partial x_{i}}+\frac{\partial}{\partial x_{j}}\left[\mu\left(\frac{\partial u_{i}}{\partial x_{j}}+\frac{\partial u_{j}}{\partial x_{i}}\right)-\rho \overline{u_{i} u_{j}}\right]+\rho g_{i}
\end{gathered}
$$

2.2 For the Reynolds stress model (RSM), the Reynolds stress transport equation is

$$
\frac{\partial}{\partial t}\left(\overline{a u_{i} u_{j}}\right)+\frac{\partial}{\partial x_{k}}\left(\rho u_{k} \overline{u_{i} u_{j}^{\prime}}\right)=P_{i j}+D_{i j}+\varphi_{i j}-\varepsilon_{i j}+F_{i j}
$$


Among them ${ }^{[5-6]}$ :

$$
\begin{aligned}
& P_{i j}=-\rho\left(\overline{u_{i}^{\prime} u_{k}^{\prime}} \frac{\partial u_{j}}{\partial x_{k}}+\overline{\overline{u_{j}^{\prime} u_{k}^{\prime}}} \frac{\partial u_{i}}{\partial x_{k}}\right) \\
& \left.D_{T i j}=-\frac{\partial}{\partial x_{k}}\left[\overline{\rho u_{i}^{\prime} u_{j}^{\prime} u_{k}^{\prime}}+\overline{p\left(\delta_{k j} u_{i}^{\prime}+\delta_{i k} u_{j}^{\prime}\right.}\right)\right] \\
& \varphi_{i j}=p\left(\overline{\frac{\partial u_{i}^{\prime}}{\partial x_{j}}+\frac{\partial u_{j}^{\prime}}{\partial x_{i}}}\right) \\
& \varepsilon_{i j}=-2 \mu \frac{\partial \frac{\partial u_{i}^{\prime}}{\partial x_{k}} \frac{\partial u_{j}^{\prime}}{\partial x_{k}}}{F_{i j}}=-2 \rho \Omega_{k}\left(\overline{u_{j}^{\prime} u_{m}^{\prime}} \varepsilon_{i k m}+\overline{u_{i}^{\prime} u_{m}^{\prime}} \varepsilon_{j k m}\right)
\end{aligned}
$$

2.3 Turbulent kinetic energy and turbulent kinetic energy equation

$$
\begin{aligned}
& \rho \frac{D k}{D t}=\frac{\partial}{\partial x_{l}}\left[\left(\frac{\mu_{t}}{\sigma_{k}}+\mu\right) \frac{\partial k}{\partial x_{l}}\right] \mu-\rho \overline{u_{i} u_{l}} \frac{\partial u_{i}}{\partial x_{l}}-\rho \varepsilon \\
& \rho \frac{D \varepsilon}{D t}=\frac{\partial}{\partial x_{l}}\left[\left(\frac{\mu_{t}}{\sigma_{\varepsilon}}+\mu\right) \frac{\partial \varepsilon}{\partial x_{l}}\right] \mu-C_{\varepsilon 1} \frac{\varepsilon}{k} \frac{\mu_{t}}{2}\left(\frac{\partial u_{i}}{\partial x_{j}}+\frac{\partial u_{j}}{\partial x_{i}}\right)^{2}-C_{\varepsilon 2} \rho \frac{\varepsilon^{2}}{k}
\end{aligned}
$$

In equations above, $\mathrm{u}$ stands for the speed of turbulent fluctuation $(\mathrm{m} / \mathrm{s})$, $\mathrm{x}$ for Descartes coordinates (m), $P_{i j}$ for generation item of stress, $D_{T i j}$ for diffusion item, $\varphi_{i j}$ for strain term of stress, $\varepsilon_{i j}$ for viscous dissipation item, and $F_{i j}$ for generation item of spinning.

\section{STRUCTURE AND OPERATIONAL PRINCIPLES OF COMPOUND HYDROCVCLONE}

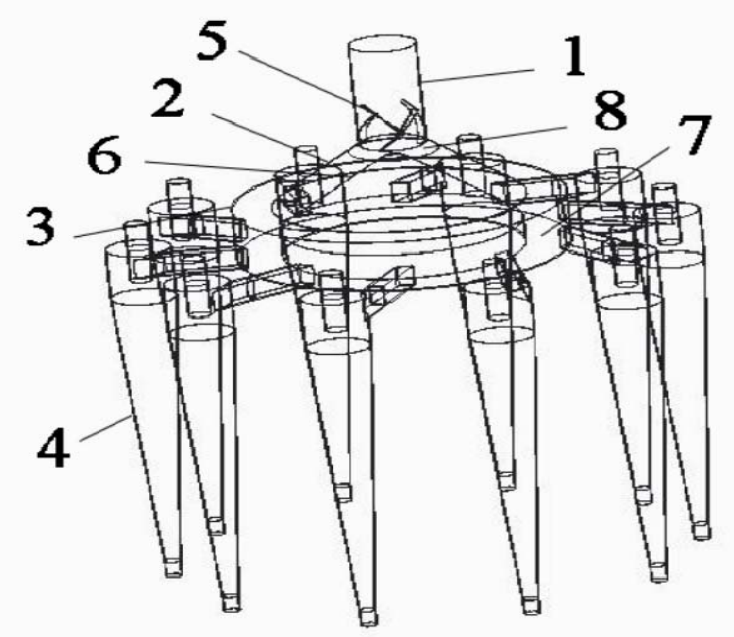

1. straight pipes 2.trumpet open pipes 3.ring pipes 4.beveling hydrocyclone with single inlet channel 5.spiral guide vanes 6.rectifiers 7.V-shaped open cavity $\quad$ 8. circular arc pipe

Figure 1. Schematic diagram of structure of compound hydrocyclone

As Figure 1 shown, the compound hydrocyclone includes two parts: flow distributor and beveling hydrocyclone with single inlet channel. Flow distributor mainly includes straight pipes, 
four spiral guide vanes, trumpet open pipes, rectifiers, ring pipes and V-shaped open cavity in front of open pipes and rectifiers. The experimental model size: the diameter of straight pipes is $102 \mathrm{~mm}$; the angle between trumpet open pipes and the horizontal surface is $40^{\circ}$; the diameter of the big circle at the bottom of open pipes is $380 \mathrm{~mm}$; the external diameter of ring pipes (whose cross section are rectangle) is $500 \mathrm{~mm}$; the helix angle and wrapping angle of spiral guide vanes are $45^{\circ}$ and $90^{\circ}$ respectively; the angle between generatrix of rectifier and horizontal plane is $45^{\circ}$. Structures of the ten beveling hydrocyclone with single inlet channel are the same as each other. The inlet channels of beveling hydrocyclone are in the shape of rectangle with length of $24 \mathrm{~mm}$ and width of $40 \mathrm{~mm}$. The diameter of the cylinder is $100 \mathrm{~mm}$ with cone angle of $8^{\circ}$. The diameter of the overflow outlet is $35 \mathrm{~mm}$ and its depth is $65 \mathrm{~mm}$. The diameter of underflow pipe is $25 \mathrm{~mm}$ and its inlet angle is $5^{\circ}$.

Through straight pipes, sewage enters flow distributors at a certain flow and pressure. With spiral guide vanes, straight flows are transformed into cyclones and enter into V-shaped open cavities at a certain speed. Finally, sewage is pressured to ten beveling hydrocyclone with single inlet channel. Sludge is concentrated through centrifugal separation. Through sludge concentration. high-concentration sewage contains $7 \%$ to $10 \%$ of solid sludge. Here, re-processing of sewage with sludge filter press or centrifuge to a large extent improves working efficiency of sludge filter press or centrifuge.

\section{NUMERICAL CALCULATION MODEL AND BOUNDARY CONDITIONS}

\subsection{Numerical calculation model}

The RNG $k-\varepsilon$ model is used for calculation of flow distributors and considers influences of spinning in flowing and cyclone flowing. The model is suitable for rapid strain, complex shearing motion of medium vortex and local transition ${ }^{[7]}$. In flow distributors, spiral flowing fits more with the RNG $k-\varepsilon$ model. Divide designed flow distributors into unstructured mesh with ICEM software. In order to improve the accuracy of calculation, the local mesh encryption is performed on the spiral flow vanes. The internal flow field of the flow distributor is calculated by using FLUENT software. The SIMPLEC algorithm is used to calculate the velocity field and pressure field, and the residual control is set to 0.000001. The pressure term is set to PRESTO algorithm and other equations use the two order upwind scheme. Figure 2(a) is a unstructured mesh model for the flow distributor.

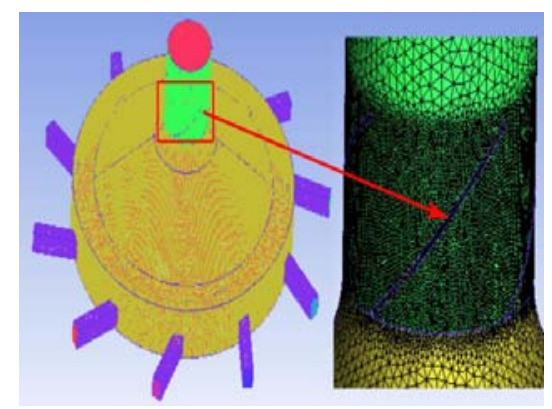

(a) Unstructured mesh model for the flow distributor.

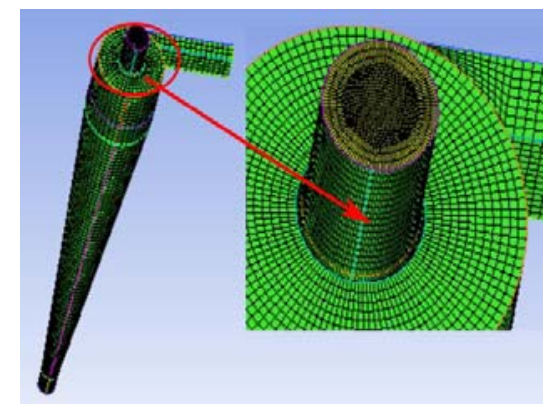

(b) Structured mesh model for the beveling hydrocyclone with single inlet channel

Figure 2. Schematic diagram of structure of the flow distributor and beveling hydrocyclone with single inlet channel 
Because of the Reynolds Stress Model $^{[8]}$ (RSM) avoiding the isotropic eddy viscosity hypothesis ,it is applied to 3D motion simulation of strong vortex flow complex. Liang Zheng et al ${ }^{[9]}$ fully explained why the Reynolds Stress Model is more suitable than other models simulating the internal flow field of hydrocyclone. The software of ICEM is utilized for hexahedral mesh generation in the hydrocyclone and the software of FLUENT for the transient calculation of flow field inside the hydrocyclone with the time step of 0.001s. The rest will be calculated with flow distributor. Figure 2(b) is a structured mesh model for beveling hydrocyclone with single inlet channel.

\subsection{Boundary conditions}

\subsubsection{Boundary conditions for flow distributor}

1) Set the inlet velocity to the $3.68 \mathrm{~m} / \mathrm{s}$ and velocity direction perpendicular to the entrance surface.

2) Each exit is used in the pressure outlet. The static pressure is $2000 \mathrm{~Pa}$.

3) No-slip boundary is adopted for the wall surface, and the standard wall function is adopted for the wall treatment.

4) Physical property parameters of the mixture: the water density is $998 \mathrm{~kg} / \mathrm{m}^{3}$ and the density of sludge is $1200 \mathrm{~kg} / \mathrm{m}^{3}$.

\subsubsection{Boundary conditions for beveling hydrocyclone with single inlet channel}

1) Set the inlet velocity to the $3.67 \mathrm{~m} / \mathrm{s}$ and velocity direction perpendicular to the entrance surface.

2)The pressure outlets are adopted as the overflow outlet and the underflow outlet, the static pressure is set as 0 and is communicated with the atmosphere.

3) Wall conditions and mixture parameters are the same as the flow distributor.

\section{RESULTS OF NUMERICAL CALCULATION}

\subsection{Analysis on the Angle of Lateral Connecting Tube of Flow Distributor}

The angel between the inlet channel of beveling hydrocyclone with single inlet channel (namely connecting tube) and tangent plane of external surface of ring pipes is different and represented as $\theta$. Relatively give values of $10^{\circ}, 20^{\circ}, 30^{\circ}, 40^{\circ}, 50^{\circ}, 60^{\circ}, 70^{\circ}, 80^{\circ}$ and $90^{\circ}$ to $\theta$. Figure $3(\mathrm{a})$ is the vertical view of flow distributor. Pressures of inlet, pressures and flows rate of 10 outlets of flow distributor are obtained under different $\theta$ through calculation of Fluent. This paper calculates pressure loss $(\Delta p)$ of models, maximal flow at outlet $Q_{\max }$ and minimal flow at outlet $Q_{\min }$. Table 1 shows performance indexes of flow distributors under different $\theta$.

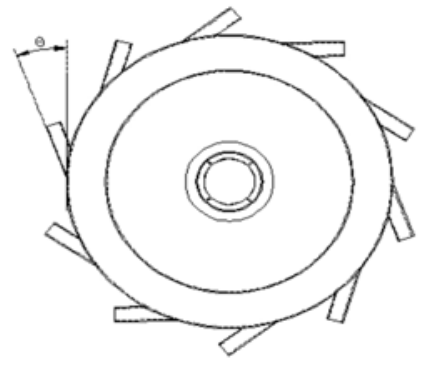

(a) The spiral guide vane is installed

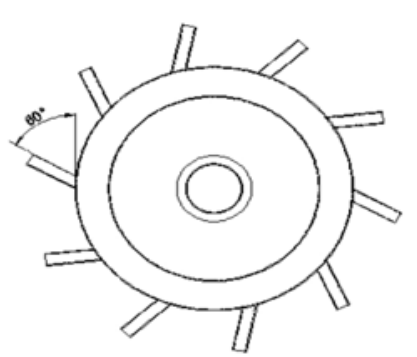

(b) The spiral guide vane is uninstalled

Figure 3. Top view of the flow distributor

As is shown in Table 1 , when $\theta$ is between $50^{\circ}$ to $70^{\circ}$, the flow distributor has lower pressure loss; When $\theta$ is between $60^{\circ}$ to $70^{\circ}$, flow distributor has relatively small maximal flow and large minimal flow at outlet. Based on comprehensive consideration, the angel between the inlet channel of beveling hydrocyclone with single inlet channel (namely connecting tube) and tangent plane of external surface of ring pipes is chosen to be $60^{\circ}$ for lower pressure drop and evener flow distribution. 
Table 1. Performance indexes of flow distributors under different $\theta$

\begin{tabular}{|c|c|c|c|c|c|c|c|c|c|}
\hline & $10^{\circ}$ & $20^{\circ}$ & $30^{\circ}$ & $40^{\circ}$ & $50^{\circ}$ & $60^{\circ}$ & $70^{\circ}$ & $80^{\circ}$ & $90^{\circ}$ \\
\hline$\Delta p(\mathrm{~Pa})$ & 19554 & 18334 & 17174 & 15462 & 15245 & 15407 & 15920 & 16027 & 16985 \\
\hline$Q_{\max }\left(m^{3} / h\right)$ & 13.06 & 12.36 & 11.18 & 10.84 & 10.99 & 10.62 & 10.80 & 10.73 & 10.82 \\
\hline$Q_{\min }\left(m^{3} / h\right)$ & 8.24 & 7.70 & 8.24 & 9.25 & 9.07 & 9.42 & 9.39 & 9.40 & 9.41 \\
\hline
\end{tabular}

\subsection{Influence of Spiral Guide Vanes on Performance of Flow Distributors}

Spiral guide vanes installed inside straight pipes can transform straight flows into rotating flows. Figure 3(b) is the vertical view of flow distributor without spiral guide vanes $\left(\theta\right.$ is $\left.60^{\circ}\right)$. Similarly, with Fluent, performance indexes with and without spiral guide vanes are figured out as is shown in Table 2. It is shown that installing spiral guide vanes can reduce pressure loss by nearly $40 \%$; compared with ones with spiral guide vanes, flow distributors without spiral guide vanes have evener flow at outlet, but the difference is not dramatic. Based on comprehensive consideration, flow distributors with spiral guide vanes are advantageous.

\begin{tabular}{|c|c|c|c|}
\hline & $\Delta p(\mathrm{~Pa})$ & $Q_{\max }\left(m^{3} / h\right)$ & $Q_{\min }\left(m^{3} / h\right)$ \\
\hline $\begin{array}{l}\text { the spiral guide vane is } \\
\text { uninstalled }\end{array}$ & 25442 & 10.22 & 9.86 \\
\hline $\begin{array}{l}\text { the spiral guide vane is } \\
\text { installed }\end{array}$ & 15407 & 10.62 & 9.42 \\
\hline
\end{tabular}

\subsection{Influence of Angle of Inlet on Performance of hydrocyclone}

\subsubsection{Influence on Velocity Field and Pressure Field}

Figure 4 shows structures of beveling hydrocyclone with single inlet channel when the angles of inlet are relatively $0^{\circ}, 5^{\circ}, 10^{\circ}$ and $15^{\circ}$. With Fluent, these 4 models are calculated. Figure 5 gives tendency of tangential velocity and axial velocity as radial position changes when cross section $\mathrm{Z}$ is $85 \mathrm{~mm}$ ( $Z$ is the distance from upper end-face of cylinder). As is shown in Figure 5(a), tendency of tangential velocity is in the shape of double peaks while axial velocity almost remains on the same level. In the zone of forced vortex, as the angle of inlet increasing, axial velocity hardly changes. In the zone of free vortex, as the angle of inlet increasing, axial velocity decreases. As is shown in Figure 5(b), as the angle of inlet increasing, axial velocity increases. The increase in axial velocity and increase in tangential velocity in the zone of free vortex show that increasing the angle of inlet within a certain range helps increase resistance and pressure loss of hydrocyclone, but the tangential velocity increasing in the zone of free vortex definitely influences separation performance of hydrocyclone and decreases their separation efficiency.

Figure 6 illustrates tendency of static pressure at the cross section $\mathrm{z}$ of $85 \mathrm{~mm}$ and $100 \mathrm{~mm}$ when the angles of inlet of beveling hydrocyclone with single inlet channel are relatively $0^{\circ}, 5^{\circ}, 10^{\circ}$ and $15^{\circ}$. It can be seen that the curves of static pressure at two cross sections also overlap with each other; on the same cross section in radial direction from the wall to central axis, static pressure decreases gradually; with increase in angle at inlet, static pressure decreases gradually. After collecting statistics on total pressure at inlet and overflow outlet, pressure loss of hydrocyclone in four different models as is shown in Table 3. It is concluded from Table 3 that when the angle of inlet is between $0^{\circ}$ and $15^{\circ}$, the bigger the angle is, the smaller the pressure loss of hydrocyclone is. 


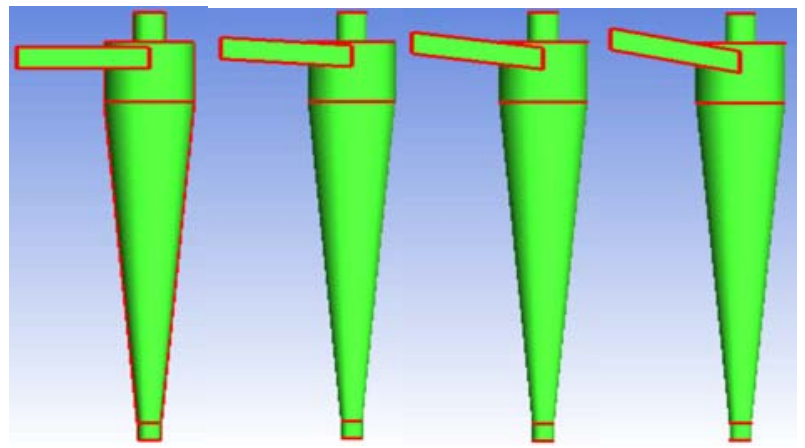
(a) $0^{\circ}$
(b) $5^{\circ}$
(c) $10^{\circ}$
(d) $15^{\circ}$

Figure 4. Structures of beveling hydrocyclone with single inlet channel when the angles of inlet are relatively $0^{\circ}, 5^{\circ}$, $10^{\circ}$ and $15^{\circ}$

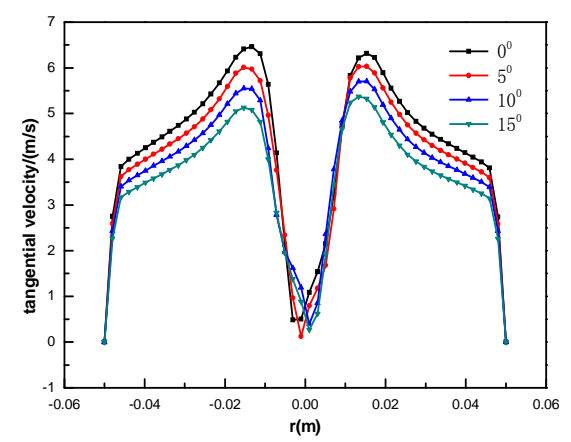

(a) Tangential velocity

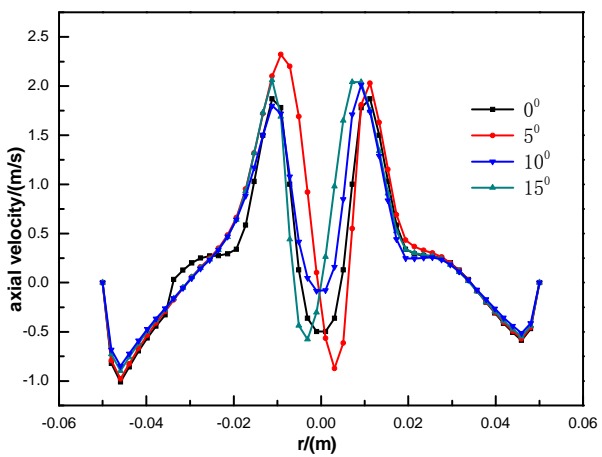

(b) Axial velocity

Figure 5. Radial distribution of tangential velocity and axial velocity of 4 different models in cross section $\mathrm{Z}=85 \mathrm{~mm}$
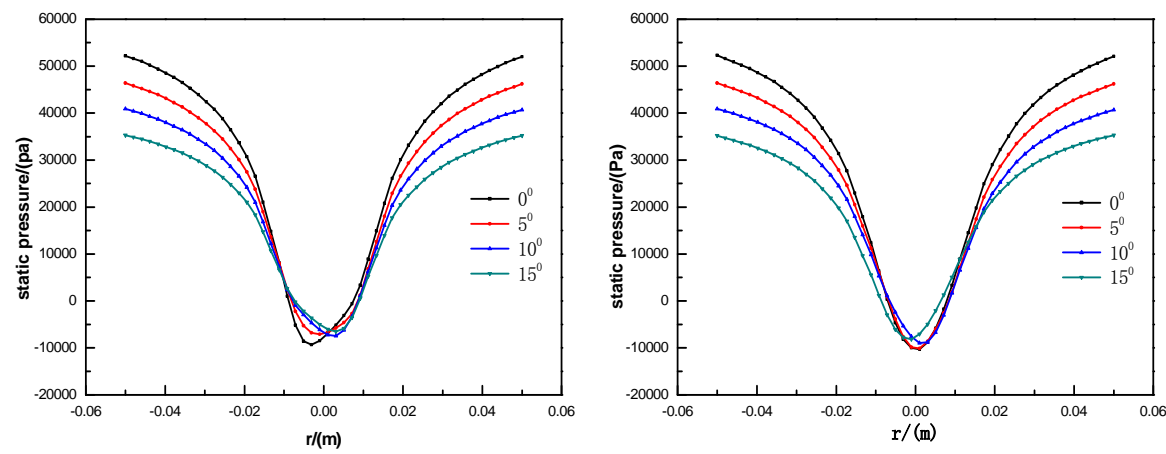

Figure 6. Radial distribution of static pressure of 4 different models in cross section $\mathrm{Z}=85 \mathrm{~mm}$ and $\mathrm{Z}=100 \mathrm{~mm}$ Table 3. pressure drops of hydrocyclone in four different models

\begin{tabular}{lllll}
\hline & $0^{\circ}$ & $5^{\circ}$ & $10^{\circ}$ & $15^{\circ}$ \\
\hline$\Delta p(\mathrm{~Pa})$ & 64764 & 54558 & 52180 & 49754
\end{tabular}

\subsubsection{Influence on Separation Efficiency}

With discrete phase model (DPM), separation efficiency of hydrocyclone with different angles of inlet is predicted. Operational and physical parameters: solid particles are sludge particles with density of $1200 \mathrm{~kg} / \mathrm{m}^{3}$; feeding flow of $0.083 \mathrm{~kg} / \mathrm{s}$ and speed at inlet of $3.67 \mathrm{~m} / \mathrm{s}$; at each time, particles of the same diameter, $10 \mu \mathrm{m}, 20 \mu \mathrm{m}, 30 \mu \mathrm{m}, 40 \mu \mathrm{m}, 50 \mu \mathrm{m}$ or $60 \mu \mathrm{m}$ are given out. Conditions of discrete phase boundary are that underflow port is set as trap and overflow port is set 
as escape, and the rest conditions of boundaries are the same as stated in chapter 4.2. After collecting information of number of particles trapped at underflow port and overflow port, separation efficiency ${ }^{[10]}$ of each diameter under different angle of inlet is figured out. Figure 7 gives separation efficiency of each sludge diameter under different angle of inlet.

According to Figure 7, as the angle of inlet increasing, separation efficiency of hydrocyclone decreases gradually; the decrease is not dramatic when the angle is between $0^{\circ}$ and $5^{\circ}$. Based on influence of angle of inlet on pressure drop stated in last chapter and on separation efficiency, the optimal angle is $5^{\circ}$.

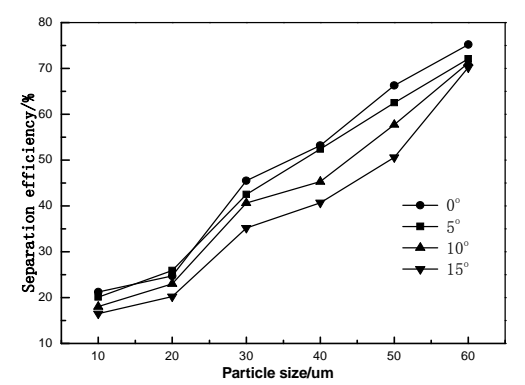

Figure 7. Separation efficiency of each sludge diameter under different angle of inlet

\section{CONCLUSION}

By means of numerical simulation, this paper imitates influence of structural parameter, spiral guide vane, and the angle of inlet of single cyclone separator on performance of compound hydrocyclone. Conclusions are obtained as follows:

1)Compared with normal hydrocyclone, the compound hydrocyclone dramatically improves processing capability and when it is used in sludge processing, it can greatly increase working efficiency of filter press or centrifuge.

2)When the angle between the inlet of beveling hydrocyclone with single inlet and the tangent plane of external surface of ring pipes is $60^{\circ}$, pressure loss is lower and flow distribution is more evener. 3)Compound hydrocyclone with spiral guide vanes saves $40 \%$ more of pressure loss than without spiral guide vanes.

4)When the angle of inlet is between $0^{\circ}$ and $15^{\circ}$, the bigger the angle is, the smaller the pressure loss and separation efficiency of hydrocyclone is. The decrease is not dramatic when the angle is between $0^{\circ}$ and $5^{\circ}$. The optimal angle is $5^{\circ}$.

\section{REFERENCES}

JANG. Ming-hu, LIU. Dao-you, ZHAO. Li-xin, JIA. Gui-chun. The Effect of Cone Angle to Hydrocyclone Pressure Field and Velocity Field[J]. Chemical Machinery, 5:572-576 (2011)

WANG. Zun-ce, GAO. Ye, Lu. Feng-xia, LIU. Xiao-ming, LI. Feng, The Effect of Liquid - liquid Hydrocyclone Inlet Structure Parameters to Pressure Characteristics[J]. Fluid Machinery, 2:16-19 (2003)

CHEN. Qi-dong, ZUO. Zhi-quan, The Flow Field Numerical Analysis of Different Lateral Inlet Cyclone[J]. China Engineering Science, 2:58-67+78 (2014)

YUAN. Hui-xin, YU. Jian-feng, LIU. Hong-bin, The Effect of Split Ratio to the Basic Performance of Oil-water Separation Hydrocyclone [J]. Petroleum Machinery, 9: 17-19+2 (2000)

YANG I H,SHIN C B,KIM T H, et al. A three-dimension simulation of a hydrocyclone for the sludge separation in water purifying plants and comparison with experimental data[J].Minerals Engineering, 2004,17:637-641.

FLUENT, User guide[R].Fluent Inc., Lebanon, NH.1998.

Zhu Hongjun, Lin Yuanhua, Xie Longhan.Fluent 12 fluid analysis and engineering simulation [M]. Beijing: Tsinghua University press, 2011.1,88.

ZHANG Shi-shuai. Computational Fluid Dynamics and the Application [M].Wuhan: Huazhong University of Science and Technology Press (2011).

LIANG. Zheng, WANG. Jin-jin, REN. Lian-cheng, LI. Lian-ming, The Theoretical Research of Solid-liquid Separation Hydrocyclone Flow Field[M]. Beijing: Petroleum Industry Press, 2:25 (2011)

Yuan Huixin. Separation process and equipment [M]. Beijing: Chemical Industry Press, 2008.1,38-42. 